\title{
Effect of Lattice Relaxations on the Hyperfine Fields of Heavy Impurities in Fe
}

\author{
T. KORHONEN ${ }^{1, \star}$, A. SETTELS ${ }^{2}$, N. PAPANIKOLAOU ${ }^{2}$, R. ZELLER ${ }^{2}$ \\ and P. H. DEDERICHS ${ }^{2}$ \\ ${ }^{1}$ Laboratory of Physics, Helsinki University of Technology, P.O.Box 1100, FIN-02015 HUT, \\ Finland; e-mail:tko@fyslab.hut.fi \\ ${ }^{2}$ Institut für Festkörperforschung, Forschungszentrum Jülich, D-52425 Jülich, Germany
}

(Received: 31 May 2000)

\begin{abstract}
We present first-principles calculations of hyperfine fields of heavy impurities in bcc Fe. In particular, the effect of lattice relaxations on the calculated hyperfine fields are studied. The calculations are based on a full-potential Korringa-Kohn-Rostoker Green's-function method for defects and employ the local spin-density approximation for the exchange and correlation effects. The calculated lattice relaxations around the $5 s p$ and $6 s p$ impurities in Fe are found to be relatively small, for the first and second nearest neighbor shells less than $6 \%$ of the nearest neighbor distance. Also the estimated relative volume changes induced by the $s p$ impurities are found to be around $60-95 \%$ of the elementary volume of Fe, surprisingly small in view of the large atomic volumes of the impurities. The calculated hyperfine fields of $5 s p$ and $6 s p$ elements are compared with available experimental data and it is shown that the inclusion of lattice relaxations in the calculation improves the agreement with experiments.
\end{abstract}

Key words: hyperfine fields, lattice distortion, dilute alloys, density functional calculations.

The hyperfine fields of impurities in the ferromagnetic hosts $\mathrm{Fe}, \mathrm{Co}$, and $\mathrm{Ni}$ have been extensively studied and a huge amount of experimental data exists $[1,2]$. The present calculations are motivated by recent measurements $[3,4]$ of the hyperfine fields of $\mathrm{Cs}$ and in particular of Fr in Fe. The measured hyperfine field of Fr is a factor of five smaller than the previous KKR calculations [5-7] predicted, which were, however, based on spherical potentials, i.e., using atomic sphere approximation (ASA), and neglected the impurity induced lattice relaxations. These lattice relaxations are in fact long ranged and lead to a volume change of the crystal. Many defect properties are influenced by these and a detailed understanding of these properties is still far from complete. In order to learn more about these effects in metals, in particular for very large impurity atoms, we present in this paper KKR calculations for impurities of the $5 s p$ and $6 s p$ series in bcc Fe for which particularly large lattice relaxations are to be expected.

\footnotetext{
^ Corresponding author.
} 
Table I. Impurity-induced volume changes in bcc Fe. Shown are the relative volume changes $\Delta V / V_{0}$ (percent of the elementary volume $V_{0}$ of $\left.\mathrm{Fe}\right)$ induced by the $5 s p$ and $6 s p$ impurities

\begin{tabular}{rrrrrrrrrrr}
\hline & $\mathrm{Cd}$ & $\mathrm{In}$ & $\mathrm{Sn}$ & $\mathrm{Sb}$ & $\mathrm{Te}$ & $\mathrm{I}$ & $\mathrm{Xe}$ & $\mathrm{Cs}$ & $\mathrm{Ba}$ & $\mathrm{La}$ \\
$\Delta V / V_{0}$ & 66 & 68 & 66 & 63 & 61 & 64 & 69 & 79 & 89 & 86 \\
& $\mathrm{Hg}$ & $\mathrm{Tl}$ & $\mathrm{Pb}$ & $\mathrm{Bi}$ & $\mathrm{Po}$ & $\mathrm{At}$ & $\mathrm{Rn}$ & $\mathrm{Fr}$ & $\mathrm{Ra}$ & $\mathrm{Ac}$ \\
$\Delta V / V_{0}$ & 72 & 84 & 87 & 88 & 90 & 89 & 93 & 96 & 96 & 91 \\
\hline
\end{tabular}

Our calculations are based on the density-functional theory with the exchange and correlation effects being treated in the local spin density approximation (LSDA) [8]. The calculations are performed using a full-potential KKR Green's-function method for defects, which is ideal for the case now studied, i.e., iron alloys in the dilute limit. The theoretical LSDA lattice constant 5.204 a.u. and an angular momentum cutoff $\ell_{\max }=4$ for the Green's function and for the radial functions are used. The potentials of five neighboring shells of Fe atoms are allowed to be perturbed, so that in total 59 perturbed potentials are recalculated self-consistently in the impurity iterations. Lattice statics methods are used to describe the long ranged relaxations and to speed up the determination of the final positions of the atoms from the ab initio forces, which are calculated using an ionic version of the Hellmann-Feynman theorem [10]. The hyperfine fields are calculated both at the ideal and the fully relaxed positions in order to study the effect of lattice relaxations on the fields. We calculate the hyperfine fields by using the relativistic generalization [11] of the contact term and neglect the orbital contribution in line with the scalar relativistic approximation used in our calculations. Since the considered $s p$ impurities have no genuine magnetic moments, the orbital contributions should be very small. Moreover the dipolar contributions vanish for the impurities due to the cubic symmetry of their surroundings.

The impurity induced lattice relaxations are found to be relatively small. For the $5 s p$ impurities the first nearest neighbor $(\mathrm{NN})$ atoms relax outwards $3.0-4.5 \%$ of the NN distance whereas for the larger $6 s p$ atoms the relaxations are somewhat larger (3.5-5.3\%). The calculated relative volume changes $\Delta V / V_{0}$ obtained from the lattice statics model are presented in Table I ( $V_{0}$ is the elementary volume of $\mathrm{Fe}$ ). Note that a $4.0 \%$ relaxation outwards corresponds to a change of the NN shell volume about $0.65 V_{0}$. These changes can also be experimentally obtained from lattice parameter measurements but for the most now studied defects the low solubility (if any) makes these measurements impossible. The largest relative volume changes are about $90 \% V_{0}$, which are really small compared to the huge atomic volumes of these impurities, which are, e.g., for Cs or Fr, up to 10-12 times larger than that of Fe. Even though the uppermost $s p$ electrons of the impurity atoms are in the conduction band of the host, the positive ions left at the lattice sites are much larger than the iron ions and in terms of elasticity language one 
Table II. The calculated hyperfine fields of $5 s p$ and $6 s p$ impurities in Fe. Shown are the full-potential KKR values calculated both at the ideal (FP ideal) and the fully relaxed (FP relax.) positions. The KKR-ASA values taken from [7] and the experimental ones from [1-4] are also shown. All values are in tesla

\begin{tabular}{lrrrrrrrrrr}
\hline & $\mathrm{Cd}$ & $\mathrm{In}$ & $\mathrm{Sn}$ & $\mathrm{Sb}$ & $\mathrm{Te}$ & $\mathrm{I}$ & $\mathrm{Xe}$ & $\mathrm{Cs}$ & $\mathrm{Ba}$ & $\mathrm{La}$ \\
Exper. & -30 & -28 & -10 & +21 & +70 & +114 & +160 & +27 & -10 & -46 \\
FP relax. & -31 & -26 & -13 & +11 & +48 & +122 & +135 & +80 & -10 & -35 \\
FP ideal & -30 & -24 & -13 & +5 & +33 & +92 & +127 & +113 & -6 & -41 \\
ASA & -39 & -34 & -21 & +3 & +36 & +78 & +128 & +160 & +4 & -43 \\
& $\mathrm{Hg}$ & $\mathrm{Tl}$ & $\mathrm{Pb}$ & $\mathrm{Bi}$ & $\mathrm{Po}$ & $\mathrm{At}$ & $\mathrm{Rn}$ & $\mathrm{Fr}$ & $\mathrm{Ra}$ & $\mathrm{Ac}$ \\
Exper. & -80 & -20 & +28 & +119 & +238 & +254 & +170 & +95 & -13 & -30 \\
FP relax. & -81 & -53 & -1 & +90 & +218 & +365 & +394 & +99 & -81 & -127 \\
FP ideal & -78 & -56 & -20 & +46 & +141 & +266 & +393 & +224 & -77 & -145 \\
ASA & -101 & -74 & -23 & +51 & +146 & +261 & +386 & +477 & -28 & -198 \\
\hline
\end{tabular}

could therefore classify the $s p$ impurities as highly compressible. This behavior is very different from the one found for $3 d$ and $4 d$ impurities in $\mathrm{Cu}$, for which the volume changes scale reasonably well with the atomic volumes of the impurities introduced into the $\mathrm{Cu}$ host by substitution.

The calculated hyperfine fields of the $5 s p$ and $6 s p$ substitutional impurities in bcc Fe are compared with experiments and earlier KKR-ASA calculations in Table II. It is seen that both the full-potential and the ASA calculations give almost identical hyperfine fields at the beginning of the series up to the rare gas atoms. Contrary to this, at the beginning of each row in the periodic table the ASA trend differs considerably from the full-potential one. This is the critical region within each row, where the hyperfine fields change dramatically between neighboring elements and where therefore numerical details of the calculations could become very important. It can also be seen from Table II that the inclusion of lattice relaxations substantially improves the agreement with the experiment. This is particularly true for the $s p$-impurities in the first half of the series, where the hyperfine field is increased due to lattice relaxations. On the other hand for the later $s p$-impurities the experimental hyperfine fields for the $s p$-impurities are not that well reproduced, especially in the $6 s p$ series there are large discrepancies for At, $\mathrm{Rn}$ as well as for Ra and Ac.

In summary, we have performed calculations of the lattice relaxations and the hyperfine fields of heavy $s p$ impurities in iron. The calculated lattice relaxations around the $5 s p$ and $6 s p$ impurities are relatively small, for the first and second $\mathrm{NN}$ shells less than $6 \%$ of the NN distance and the volume change induced by these impurities was found to be around $60-95 \%$ of the elementary volume of Fe. The calculated hyperfine fields of $5 s p$ and $6 s p$ substitutional impurities reproduce the experimentally observed trend qualitatively well and also the quantitative agree- 
ment is good for the most of the impurities. The inclusion of lattice relaxations in the calculations was also seen to improve the overall agreement of the calculated results with experiments.

\section{Acknowledgements}

This work was supported by a bilateral German-Greek cooperation grant and was partially funded by the TMR Network "Interface Magnetism" (Contract: ERBFMRXCT960089).

\section{References}

1. Krane, K. S., Hyp. Interact. 15/16 (1983), 1069.

2. Rao, G. N., Hyp. Interact. 24/25 (1985), 1119.

3. Ashworth, C. J., Back, P., Ohya, S., Stone, N. J. and White, J. P., Hyp. Interact. 59 (1990), 461.

4. Will, B., Herzog, P., Paulsen, R., Camps, J., De Moor, P., Schuurmans, P., Severijns, N., Van Geert, A., Vanneste, L., Berkes, I., De Jesus, M., Lindroos, M. and Van Duppen, P., Phys. Rev. B 57 (1998), 11527.

5. Akai, H., Akai, M., Blügel, S., Zeller, R. and Dederichs, P. H., J. Magn. Magn. Mater. 45 (1984), 291.

6. Akai, M., Akai, H. and Kanamori, J., J. Phys. Soc. Japan 54 (1985), 4246, 4257; 56 (1987), 1064.

7. Akai, H., Akai, M., Blügel, S., Drittler, B., Ebert, H., Terakura, K., Zeller, R. and Dederichs, P. H., Progr. Theoret. Phys. Suppl. 101 (1990), 11.

8. Vosko, S. H., Wilk, L. and Nusair, M., Canad. J. Phys. 58 (1980), 1200.

9. Ceperley, D. M. and Alder, B. J., Phys. Rev. Lett. 45 (1980), 566.

10. Papanikolaou, N., Zeller, R., Dederichs, P. H. and Stefanou, N., Phys. Rev. B 55 (1997), 4157.

11. Blügel, S., Akai, H., Zeller, R. and Dederichs, P. H., Phys. Rev. B 35 (1987), 3271. 